\title{
Fourier coefficients of real analytic cusp forms of arbitrary real weight
}

\author{
by \\ Roland Matthes (Kassel)
}

1. Introduction. The main purpose of this paper is to give a mean square estimate for the Fourier coefficients of vector-valued real analytic modular cusp forms of arbitrary real weight. For the case of holomorphic forms such estimates were given by Rankin [6] and Selberg [9]. Let $a(n)$ be the $n$th Fourier coefficient of a holomorphic modular cusp form of weight $r>0$. Then their result reads

$$
\sum_{n \leq x} \frac{|a(n)|^{2}}{n^{r-1}}=A x+\mathcal{O}\left(x^{3 / 5}\right)
$$

and it is derived from the analytic properties of the Dirichlet series

$$
R(s)=\sum_{n>0} \frac{|a(n)|^{2}}{n^{s+r-1}},
$$

called the Rankin-Selberg zeta function, using a theorem of Landau (see [2]). This Dirichlet series arises when integrating $y^{r}$ times the square of the holomorphic cusp form against the real analytic Eisenstein series of weight zero over a fundamental domain. The analytic properties of $R(s)$, such as a functional equation, are inherited from those of the Eisenstein series.

When turning to real analytic cusp forms, also nonvanishing Fourier coefficients at negative indexes occur and we are faced with two Dirichlet series, $R_{+}(s)$ and $R_{-}(s)$ (see Section 2 for the definition), either of which longs for analytic continuation.

In a previous paper, [4], we showed how to achieve an analytic continuation of both Dirichlet series by slightly modifying the Rankin-Selberg method. This already gives a mean square formula of the desired form, but with a bad error term. In order to obtain an error term as good as in the holomorphic case one has to perform some tedious estimates.

Supported by a grant of the DFG. 
The crucial point here is the investigation of the Mellin transform

$$
\mathcal{M}_{\kappa, i t}(s)=\int_{0}^{\infty} W_{\kappa, i t}^{2}(y) y^{s-2} d y, \quad \kappa \text { real, } \operatorname{Re}(s)>|2 \operatorname{Re}(i t)|,
$$

where $W_{\kappa, i t}$ is the exponentially decreasing Whittaker function. Here $\kappa$ corresponds to the weight and the parameter $t$ is intimately related to the spectrum of the Laplace-Beltrami operator. For weight zero, i.e. $\kappa=0$, $\mathcal{M}_{\kappa, i t}(s)$ reduces to a $\Gamma$-factor, which can be handled via Stirling's formula.

In [4] we proved the recurrence relation

$$
\text { (2) }(s+1) \mathcal{M}_{\kappa, i t}(s+2)-2 \kappa(2 s+1) \mathcal{M}_{\kappa, i t}(s+1)=s\left(s^{2}+4 t^{2}\right) \mathcal{M}_{\kappa, i t}(s) \text {, }
$$

from which it follows that $\mathcal{M}_{\kappa, i t}(s)$ is a meromorphic function with simple poles at $s=0,-1, \ldots$ and $s= \pm 2 i t,-1 \pm 2 i t, \ldots$

In our present paper we shall investigate the asymptotic behaviour of $\mathcal{M}_{\kappa, i t}(s)$ for $\operatorname{Im}(s) \rightarrow \infty$ (see Lemma 1 below), which is sufficient for our purposes. It also enables us to give a growth estimate for our Rankin-Selberg zeta functions in the critical strip.

We tried to keep our estimates uniform with respect to the spectral parameter $t_{k}$.

In [5] we use Theorem 1 of the present paper for improving the error term in the prime geodesic theorem for half-integral weights.

2. Statement of results. Let $\Xi$ be a unitary $m$-dimensional multiplier system of weight $r$ for $\Gamma:=\mathrm{SL}(2, \mathbb{Z})$ and $\vec{f}_{1}, \ldots, \vec{f}_{m}$ be an orthonormal set of eigenvectors of $\Xi\left(\left(\begin{array}{ll}1 & 1 \\ 0 & 1\end{array}\right)\right)$ with eigenvalues $e^{2 \pi i \alpha_{j}}, 0 \leq \alpha_{j}<1, j=1, \ldots, m$. A real analytic automorphic form for $\Gamma$ with respect to $\Xi$ is a function $F(z)$ on the upper half-plane $\mathcal{H}=\{z \in \mathbb{C} \mid \operatorname{Im}(z)>0\}$ with values in $\mathbb{C}^{m}$ which satisfies

(i) $F(M z)=\Xi(M) e^{i r \arg (c z+d)} F(z), M=\left(\begin{array}{ll}a & b \\ c & d\end{array}\right) \in \Gamma$,

(ii) $-\Delta_{r} F=\lambda F$, where $\Delta_{r}=y^{2}\left(\frac{\partial^{2}}{\partial x^{2}}+\frac{\partial^{2}}{\partial y^{2}}\right)-i r y \frac{\partial}{\partial x}$ is the so-called Laplace-Beltrami operator,

(iii) $F(z)=\mathcal{O}\left(y^{c}\right)$ for $y \rightarrow \infty$ with some constant $c>0$.

Automorphic forms as just defined are eigenfunctions of an elliptic differential operator and hence are real analytic functions.

Denote by $L^{2}(\Gamma \backslash \mathcal{H}, r, \Xi)$ the Hilbert space of functions $F: \mathcal{H} \rightarrow \mathbb{C}^{m}$ which satisfy the automorphy condition (i) and are square-integrable, i.e.

$$
\int_{D_{\Gamma}}|F(z)|^{2} \frac{d x d y}{y^{2}}<\infty
$$

where $D_{\Gamma}$ is a fundamental domain for $\Gamma$. It is well known (see [7], [8]), that the Laplace-Beltrami operator has a self-adjoint extension in $L^{2}(\Gamma \backslash \mathcal{H}, r, \Xi)$, 
the spectrum being contained in $[|r| / 2(1-|r| / 2), \infty)$. This gives rise to the spectral resolution

$$
L^{2}(\Gamma \backslash \mathcal{H}, r, \Xi)=\mathbb{C} \oplus L_{\text {disc }}^{2}(\Gamma \backslash \mathcal{H}, r, \Xi) \oplus L_{\text {cont }}^{2}(\Gamma \backslash \mathcal{H}, r, \Xi),
$$

where the constant functions only occur for $r=0$ and the continuous part of the spectrum is present only if $\Xi$ becomes singular, i.e. if some $\alpha_{j}$ vanishes.

The space $L_{\text {disc }}^{2}(\Gamma \backslash \mathcal{H}, r, \Xi)$ is spanned by real analytic automorphic forms. Let $\left\{u_{k}\right\}$ be an orthonormal basis for $L_{\text {disc }}^{2}(\Gamma \backslash \mathcal{H}, r, \Xi)$. In the sequel we shall especially be interested in the subspace of cusp forms, i.e. forms that are of exponential decay at infinity, an orthonormal basis $\left\{u_{k}\right\}=: \mathcal{C}_{r, \Xi}$ of which is chosen once for the rest of these notes.

It is convenient to write the eigenvalue of $u_{k}$ in the form

$$
\lambda_{k}=\left(\frac{1}{2}+i t_{k}\right)\left(\frac{1}{2}-i t_{k}\right)
$$

with $t_{k} \geq 0$ for $\lambda_{k} \geq 1 / 4$ and $t_{k}$ purely imaginary with $\operatorname{Im}\left(t_{k}\right)<0$ if $\lambda_{k}<1 / 4$.

Any $u_{k} \in \mathcal{C}_{r, \Xi}$ has a Fourier expansion at infinity (cf. [7])

$$
u_{k}(z)=\sum_{j=1}^{m} \psi_{j}(z) \vec{f}_{j}
$$

with

$$
\psi_{j}(z)=\sum_{\substack{n \equiv \alpha_{j} \bmod 1 \\ n \neq 0}} \varrho_{j, k}(n) W_{\operatorname{sgn}(n) r / 2, i t_{k}}(4 \pi|n| y) e^{2 \pi i n x} .
$$

Definition. Let $u_{k} \in \mathcal{C}_{r, \Xi}$. The Rankin-Selberg zeta functions belonging to $u_{k}$ are given by the Dirichlet series

$$
R_{ \pm, k}(s)=\sum_{j=1}^{m} \sum_{\substack{n \equiv \alpha_{j} \bmod 1 \\ \pm n>0}} \frac{\left|\varrho_{j, k}(n)\right|^{2}}{|n|^{s-1}} .
$$

In [4] we showed that it is sufficient to restrict oneself to $r \in[-1,1)$, since all possible Rankin-Selberg zeta functions already occur for these weights. We now state the main result of [4], where we write for short $\mathcal{M}_{ \pm, k}(s)$ instead of $\mathcal{M}_{ \pm r / 2, i t_{k}}(s)$ :

Proposition 1. Let $u_{k} \in \mathcal{C}_{r, \Xi}$ with $r \in[-1,1)$.

(i) The abscissas of convergence of $R_{ \pm, k}(s)$ are 1 . Moreover, we can continue $\zeta(2 s) R_{ \pm, k}(s)$ as meromorphic functions over the whole complex plane with the only pole at $s=1$. Denote the residues at $s=1$ by $b_{ \pm, k}$ and put

$$
\mathbf{M}_{k}(s)=\left(\begin{array}{cc}
\mathcal{M}_{-, k}(s+1) & \mathcal{M}_{-, k}(s) \\
\mathcal{M}_{+, k}(s+1) & -\mathcal{M}_{+, k}(s)
\end{array}\right)
$$


Then

$$
b_{ \pm, k}=\frac{3\left(\mathcal{M}_{\mp, k}(2) \pm r \mathcal{M}_{\mp, k}(1)\right.}{\pi \operatorname{det} \mathbf{M}_{k}(1)} .
$$

(ii) We write

and have a functional equation

$$
\mathbf{R}_{k}(s)=\left(\begin{array}{c}
R_{+, k}(s) \\
R_{-, k}(s)
\end{array}\right)
$$

$$
\zeta(2 s) \mathbf{R}_{k}(s)=\mathbf{C}_{k}(s) \zeta(2-2 s) \mathbf{R}_{k}(1-s)
$$

with

$$
\mathbf{C}_{k}(s)=\frac{4^{s} \pi^{4 s-3 / 2} \Gamma(1-s) \Gamma(2 s-1)}{\Gamma^{2}(s) \Gamma\left(s-\frac{1}{2}\right) \operatorname{det} \mathbf{M}_{k}(s)}\left(\begin{array}{cc}
c_{-+, k}(s) & c_{--, k}(s) \\
c_{++, k}(s) & c_{+-, k}(s)
\end{array}\right),
$$

where

(3) $\quad c_{x y, k}(s)=\mathcal{M}_{x, k}(s+1) \mathcal{M}_{y, k}(1-s)+\delta(x, y)\left(s^{2}+4 t_{k}^{2}\right) \mathcal{M}_{x, k}(s) \mathcal{M}_{y, k}(-s)$ and $\delta(x, y)=1$ if $x=y$ and -1 otherwise.

We are now in a position to state the main results of our paper.

Theorem 1. Put $R_{ \pm, k}^{*}(s):=\zeta(2 s) R_{ \pm, k}(s)$ and $Q_{k}:=\max \left(2\left|t_{k}\right|, 1\right)$. Then for small $\delta>0, s=\sigma+i \tau$ with $-\delta<\sigma<1+\delta$ and all $u_{k} \in \mathcal{C}_{r, \Xi}$ with $r \in[-1,1)$ we have

$$
(s-1) R_{ \pm, k}^{*}(s) \ll \delta^{-2}|\tau|^{-2 \sigma+3+2 \delta} Q_{k}^{-\sigma+1+\delta} S_{ \pm, k}(1+\delta), \quad|\tau| \rightarrow \infty,
$$

where

$$
S_{ \pm, k}(1+\delta):=\max \left(R_{ \pm, k}(1+\delta), Q_{k}^{\mp 2 r} R_{\mp, k}(1+\delta)\right)
$$

and the implied constant depends neither on $\sigma$ nor on $k$.

TheOREM 2. Choose $x>0$ such that $x / d \not \equiv \alpha_{j} \bmod 1$ for any positive integer $d \leq x$. We then define

$$
A_{ \pm, k}(x):=\sum_{j=1}^{m} \sum_{\substack{n \equiv \pm \alpha_{j} \bmod 1 \\ 0<n \leq x}}\left|\varrho_{j, k}( \pm n)\right|^{2} n
$$

and obtain for small $\varepsilon>0$

$$
A_{ \pm, k}(x)=b_{ \pm, k} x+\mathcal{O}_{\varepsilon}\left(Q_{k}^{3 / 2+\varepsilon} x^{3 / 5+\varepsilon} S_{ \pm, k}\left(1+\frac{1}{\log x Q_{k}}\right)\right), \quad x \rightarrow \infty,
$$

with the implied constant depending on $\varepsilon$ alone.

\section{Tedious but useful}

Lemma 1. Let $-1 / 2 \leq \kappa<1 / 2, t>0$ or $t$ purely imaginary with $|\kappa|-1 / 2<\operatorname{Im}(t) \leq 0$. Write $s=\sigma+i \tau$ and put $Q:=\max (2|t|, 1)$. 
(i) If $|\operatorname{Im}(t)|<\sigma \leq 1000$ and $|\tau| \geq Q$, then

$$
\mathcal{M}_{\kappa, i t}(s)=\frac{\Gamma\left(s-\frac{1}{2}+\kappa-i t\right) \Gamma\left(s-\frac{1}{2}+\kappa+i t\right)}{\Gamma(s)}\left(1+\mathcal{O}\left(|\tau|^{-1 / 2} Q\right)\right) .
$$

(ii) For small $\delta>0$ and $\delta \leq \sigma \leq 1000,|\tau| \leq Q$ and $t>0$, we have

$$
\mathcal{M}_{\kappa, i t}(s) \ll \delta^{-1} e^{-\pi t} Q^{\sigma-1+2 \kappa} .
$$

Remark. For two functions $f, g: D \subset \mathbb{C}^{n} \mapsto \mathbb{C}^{m}$ the notion $f \ll g$ or $f=\mathcal{O}(g)$ shall mean that there is a positive constant $M$ such that $\left|f\left(z_{1}, \ldots, z_{n}\right)\right| \leq M\left|g\left(z_{1}, \ldots, z_{n}\right)\right|$ on $D$. In our situation think of $\mathcal{M}_{\kappa, i t}(s)$ as $\mathcal{M}(\kappa, t, s)$.

Pro of of Lemma 1. First observe that $W_{\kappa, i t}(y)$ is real for our choice of the parameters, hence $\mathcal{M}_{\kappa, i t}(\bar{s})=\overline{\mathcal{M}_{\kappa, i t}(s)}$. So we may restrict ourselves to positive $\tau$. In [4] we have proved

$\mathcal{M}_{\kappa, i t}(s)$

$$
=\frac{1}{2 \pi i} \int_{L} \frac{\begin{array}{c}
\Gamma\left(-\frac{1}{2}+v+s+i t\right) \Gamma\left(-\frac{1}{2}+v+s-i t\right) \\
\times \Gamma\left(\frac{1}{2}-v+i t\right) \Gamma\left(\frac{1}{2}-v-i t\right) \Gamma(v-\kappa)
\end{array}}{\Gamma(v+s-\kappa) \Gamma\left(\frac{1}{2}-\kappa+i t\right) \Gamma\left(\frac{1}{2}-\kappa-i t\right)} d v
$$

where $L$ runs from $-i \infty$ to $+i \infty$ and has to be chosen such that all poles of $\Gamma(v-\kappa)$ are to the left and all poles of $\Gamma\left(\frac{1}{2}-v+i t\right)$ and $\Gamma\left(\frac{1}{2}-v-i t\right)$ are to the right of $L$. This formula is valid for all $s \in \mathbb{C}$ for which $\operatorname{Re}\left(v+s-\frac{1}{2}\right)>0$ for all $v \in L$. If we choose for $L$ the line $\operatorname{Re}(v)=\frac{1}{2}+\operatorname{Im}(t)$, avoiding the poles at $\frac{1}{2} \pm i t$ by small semicircles, we see that our above representation is valid for $\sigma>|\operatorname{Im}(t)|$.

Now for real $t$ we replace $L$ by either $L_{1}$ or $L_{2}$ or $L_{3}$ (see Fig. 1), subject to $s \in S_{1}, S_{2}, S_{3}$, where $S_{1}=\{s \in \mathbb{C}: 0<\sigma \leq 1-\kappa, Q \leq \tau<2 Q\}, S_{2}=$ $\{s \in \mathbb{C}: 0<\sigma \leq 1-\kappa, 2 Q \leq \tau\}, S_{3}=\{s \in \mathbb{C}: 1-\kappa<\sigma \leq 1000, Q \leq \tau\}$.

$L_{1}$ consists of three segments

$$
\begin{gathered}
L_{11}=\left[\kappa-\frac{1}{2}-i \delta_{t}, \kappa-\frac{1}{2}+i \infty\right), \quad L_{12}=\left[\kappa-\frac{1}{2}-i \delta_{t}, 1-\sigma-i \delta_{t}\right], \\
L_{13}=\left[1-\sigma-i \delta_{t}, 1-\sigma-i \infty\right],
\end{gathered}
$$

with $\delta_{t}=1 / 4$ if $t \geq 1 / 2$, and $\delta_{t}=3 / 4$ if $t<1 / 2$. Similarly, $L_{2}$ consists of

$$
\begin{gathered}
L_{21}=\left[\kappa-\frac{1}{2}-\frac{i \tau}{2}, \kappa-\frac{1}{2}+i \infty\right), \quad L_{22}=\left[\kappa-\frac{1}{2}-\frac{i \tau}{2}, 1-\sigma-\frac{i \tau}{2}\right], \\
L_{23}=\left[1-\sigma-\frac{i \tau}{2}, 1-\sigma-i \infty\right],
\end{gathered}
$$

while for $L_{3}$ we choose the line $\operatorname{Re}(v)=\kappa-\frac{1}{2}$. If $t$ is purely imaginary, then in either case we replace $L$ by $L_{3}$. 

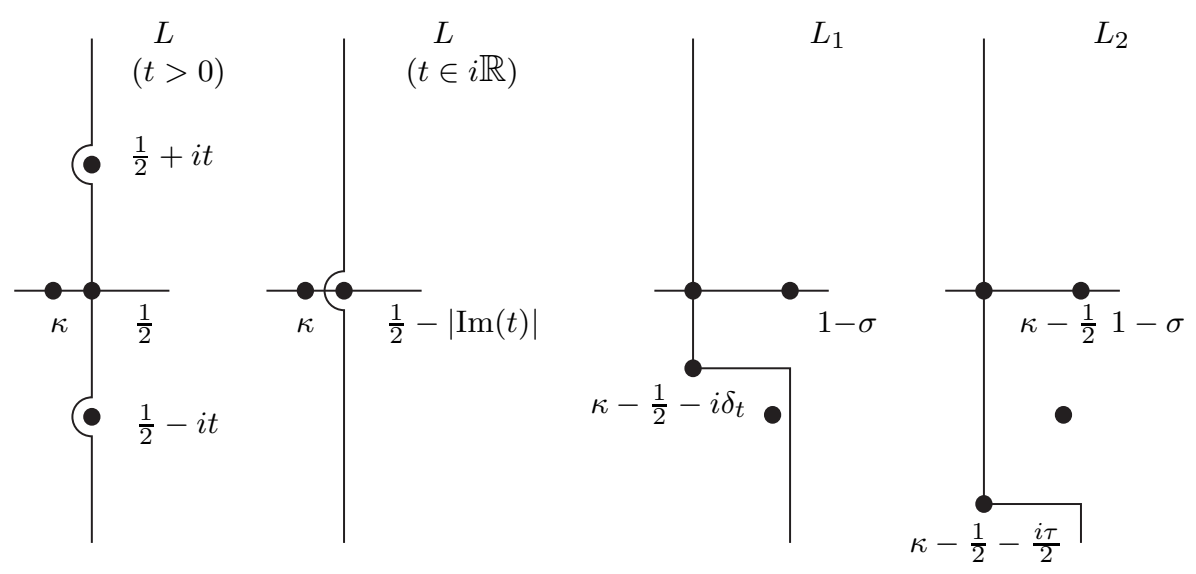

Fig. 1

Notice that in either case we pass a pole at $v=\kappa$ and if $\sigma<\frac{1}{2}$ and $s \in S_{1}$ there is a further pole at $v=\frac{1}{2}-i t$. If $\sigma=\frac{1}{2}$ we avoid this pole by a small semicircle as before. The corresponding contributions to the integral are

$$
\begin{aligned}
R_{\kappa}(s, t) & =\frac{\Gamma\left(s-\frac{1}{2}+\kappa-i t\right) \Gamma\left(s-\frac{1}{2}+\kappa+i t\right)}{\Gamma(s)}, \\
R_{1 / 2-i t}(s, t) & =\frac{\Gamma(s) \Gamma(s-2 i t) \Gamma(2 i t)}{\Gamma\left(s+\frac{1}{2}-\kappa-i t\right) \Gamma\left(\frac{1}{2}-\kappa+i t\right) \Gamma\left(\frac{1}{2}-\kappa-i t\right)} .
\end{aligned}
$$

We do not pass poles of $\Gamma\left(-\frac{1}{2}+v+s \pm i t\right)$ because of our choice of $L_{i}$ and $S_{i}, i=1,2,3$.

The case $t>0$. We utilize Stirling's formula for the $\Gamma$-function

$$
\Gamma(s)=\sqrt{\frac{2 \pi}{s}} e^{s(\log s-1)}\left(1+\mathcal{O}\left(|s|^{-1}\right)\right), \quad|\arg s|<\pi,
$$

giving

$$
|\Gamma(s)|=\sqrt{2 \pi}|s|^{\sigma-1 / 2} e^{-\tau \arg s-\sigma}\left(1+\mathcal{O}\left(|s|^{-1}\right)\right), \quad|\arg s|<\pi,
$$

in order to obtain for $s \in S_{i}$ (writing $v=\nu+i \varrho$ )

(5) $\mathcal{M}_{\kappa, i t}(s)$

$$
\begin{array}{r}
=R_{\kappa}(s, t)+\left\{\text { only if } \sigma<\frac{1}{2} \text { and } Q \leq \tau<2 Q\right\} R_{1 / 2-i t}(s) \\
+\mathcal{O}\left(Q^{2 \kappa} e^{\pi t} \int_{L_{i}} \frac{\left|s+v i-\frac{1}{2}+i t\right|^{\sigma+\nu-1}\left|s+v-\frac{1}{2}-i t\right|^{\sigma+\nu-1}|v-\kappa|^{\nu-\kappa-1 / 2}}{|s+v-\kappa|^{\sigma+\nu-\kappa-1 / 2}\left|\frac{1}{2}-v-i t\right|^{\nu}\left|\frac{1}{2}-v+i t\right|^{\nu}}\right. \\
\left.\times e^{-\sigma-f(\varrho, \tau, t, \sigma, \nu, \kappa)} d v\right)
\end{array}
$$


with

$$
\begin{aligned}
& f(\varrho, \tau, t, \sigma, \nu, \kappa) \\
& =A\left(\varrho+\tau+t, \sigma+\nu-\frac{1}{2}\right)+A\left(\varrho+\tau-t, \sigma+\nu-\frac{1}{2}\right)+A(\varrho, \nu-\kappa) \\
& \quad+A\left(\varrho+t, \frac{1}{2}-\nu\right)+A\left(t-\varrho, \frac{1}{2}-\nu\right)-A(\varrho+\tau, \sigma+\nu-\kappa),
\end{aligned}
$$

where

$$
A(x, y)= \begin{cases}x \arctan \frac{x}{y} & \text { if } y>0 \\ |x| \frac{\pi}{2} & \text { if } y=0 \\ |x|\left(\pi-\arctan \frac{|x|}{|y|}\right) & \text { if } y<0 .\end{cases}
$$

Notice that we can indeed choose the implied constant in (5) to be absolute. To see this, one should observe that the implied constant in (4) is absolute for $\operatorname{Re}(s)>1$ (see e.g. [3]). In our situation the real part in the argument of the $\Gamma$-factors might be smaller than 1 . But then we just use the functional equation $s \Gamma(s)=\Gamma(s+1)$ a finite number of times.

Now there is a constant $M>0$ such that for any $x \in \mathbb{R}$ and $y \leq 1000$ we have

$$
\left|A(x, y)-\frac{|x| \pi}{2}\right|<M
$$

We still define

$$
g(\varrho, \tau, t):= \begin{cases}\pi(2 \varrho+\tau / 2) & \text { if } t<\varrho \\ \pi(\varrho+t+\tau / 2) & \text { if } 0<\varrho \leq t \\ \pi(t+\tau / 2) & \text { if }-t<\varrho \leq 0 \\ \pi(|\varrho|+\tau / 2) & \text { if }-\tau+t<\varrho \leq-t \\ \pi(2|\varrho|+t-\tau / 2) & \text { if }-\tau<\varrho \leq-\tau+t \\ \pi(|\varrho|+t+\tau / 2) & \text { if }-\tau-t<\varrho \leq-\tau \\ \pi(2|\varrho|-\tau / 2) & \text { if } \varrho \leq-\tau-t\end{cases}
$$

Then we see that there is a positive constant $M^{*}$ such that

$$
|f(\varrho, \tau, t, \sigma, \nu, \kappa)-g(\varrho, \tau, t)|<M^{*}
$$

for all $s \in S_{i}, v \in L_{i}, t>0$ and $\kappa \in\left[-\frac{1}{2}, \frac{1}{2}\right)$. This enables us to estimate the integral in the $\mathcal{O}$-term in (5). For $s \in S_{1}$ we obtain

$$
\begin{aligned}
\int_{L_{11}} & \ll \int_{-1}^{\infty} \frac{|1+i(\tau+t+\varrho)|^{\sigma+\kappa-3 / 2}|1+i(\tau-t+\varrho)|^{\sigma+\kappa-3 / 2} e^{-g(\varrho, \tau, t)}}{|1+i(\tau+\varrho)|^{\sigma-1}|1+i(\varrho+t)|^{\kappa-1 / 2}|1+i(\varrho-t)|^{\kappa-1 / 2}|1+i \varrho|} d \varrho \\
& \ll \tau^{-2+2 \kappa+\sigma} Q^{-2 \kappa+1} e^{-\pi(\tau / 2+t)} .
\end{aligned}
$$

The same estimate holds for $\int_{L_{12}}$. Notice that we used $\frac{1}{2}-\kappa>0$. Further- 
more,

$$
\begin{aligned}
\int_{L_{13}} & \ll \int_{0}^{\infty} \frac{|1+i \varrho|^{1 / 2-\sigma-\kappa} e^{-g(-\varrho, \tau, t)}}{|1+i(\tau-\varrho)|^{1 / 2-\kappa}|1+i(\varrho+t)|^{1-\sigma}|1+i(\varrho-t)|^{1-\sigma}} d \varrho \\
\ll & \tau^{\kappa-1 / 2} e^{-\pi(\tau / 2+t)}\left(\int_{0}^{t} \frac{(1+\varrho)^{1 / 2-\sigma-\kappa}}{|1+i(\varrho+t)|^{1-\sigma}|1+i(\varrho-t)|^{1-\sigma}} d \varrho\right. \\
& \left.+\int_{t}^{\infty} \frac{(1+\varrho)^{1 / 2-\sigma-\kappa} e^{-\pi(\varrho-t)}}{|1+i(\varrho+t)|^{1-\sigma}|1+i(\varrho-t)|^{1-\sigma}} d \varrho\right) \\
\ll & \tau^{\kappa-1 / 2} Q^{\sigma-1} e^{-\pi(\tau / 2+t)}\left(\int_{0}^{t} \frac{(1+\varrho)^{1 / 2-\sigma-\kappa}}{(1+t-\varrho)^{1-\sigma}} d \varrho\right. \\
& \left.+\int_{t}^{\infty} \frac{(1+\varrho)^{1 / 2-\sigma-\kappa} e^{-\pi(\varrho-t)}}{(1+\varrho-t)^{1-\sigma}} d \varrho\right) .
\end{aligned}
$$

It is seen at once that the second integral is $\ll Q^{1 / 2-\sigma-\kappa}$. The first integral is majorized by

$$
\begin{aligned}
\int_{0}^{t+2} \varrho^{1 / 2-\sigma-\kappa}(t+2- & \varrho)^{\sigma-1} d \varrho \\
& \ll Q^{1 / 2-\kappa} \int_{0}^{1} \varrho^{1 / 2-\sigma-\kappa}(1-\varrho)^{\sigma-1} d \varrho \ll Q^{1 / 2-\kappa},
\end{aligned}
$$

since $0<\sigma<3 / 2-\kappa$. Now, putting everything together and recalling that $Q \leq \tau<2 Q$, we obtain

$$
\mathcal{M}_{\kappa, i t}(s)=R_{\kappa}(s, t)+R_{1 / 2-i t}(s, t)+\mathcal{O}\left(\tau^{\sigma-2+2 \kappa} Q e^{-\pi \tau / 2}\right) .
$$

We still observe that from (4) it follows that for $s \in S_{1}$ and $t>0$,

$$
R_{\kappa}(s, t) \gg \tau^{\sigma-3 / 2+2 \kappa} e^{-\pi \tau / 2}, \quad R_{1 / 2-i t}(s, t) \ll \tau^{\sigma-1 / 2+\kappa} Q^{\kappa-1 / 2} e^{-\pi \tau / 2},
$$

which concludes the proof for $t>0$ and $s \in S_{1}$. The proof for the remaining cases is similar.

For the proof of (ii) we use the following formulas, which we proved in [4]:

$$
\mathcal{M}_{\kappa, i t}(1)=\frac{2 \pi \operatorname{Im}\left(\Psi\left(\frac{1}{2}-\kappa+i t\right)\right)}{\left|\Gamma\left(\frac{1}{2}-\kappa-i t\right)\right|^{2} \operatorname{sh} 2 \pi t}
$$

and

$$
\begin{array}{r}
\mathcal{M}_{\kappa, i t}(2)=\frac{(2-4 \kappa) \pi t^{3}}{\left|\Gamma\left(\frac{1}{2}-\kappa-i t\right)\right|^{2} \operatorname{sh} 2 \pi t}\left(\frac{1}{\left(\frac{1}{2}+\kappa\right)^{2}+t^{2}}+\frac{\Psi^{\prime}(-2 i t)-\Psi^{\prime}(2 i t)}{4 i t}\right) \\
+\mathcal{O}\left(e^{-\pi t} t^{2 \kappa} \log t\right), \quad t \rightarrow \infty,
\end{array}
$$


with $\Psi$ the logarithmic derivative of the $\Gamma$-function. We notice that

$$
\Psi(z)=\log z+\mathcal{O}(1 /|z|), \quad|z| \rightarrow \infty
$$

(cf. [1], p. 75). Furthermore, we use

$$
\Psi^{\prime}(z)=\sum_{n=0}^{8} \frac{1}{(z+n)^{2}}
$$

(cf. [3], p. 14), giving

$$
\Psi^{\prime}( \pm 2 i t)=\sum_{n=0}^{\infty} \frac{n^{2}-4 t^{2} \mp 4 i n t}{\left(4 t^{2}+n^{2}\right)^{2}} .
$$

Hence

$$
\begin{aligned}
\frac{\Psi^{\prime}(-2 i t)-\Psi^{\prime}(2 i t)}{4 i t} & =\sum_{n=0}^{\infty} \frac{2 n}{\left(4 t^{2}+n^{2}\right)^{2}} \\
& =\int_{0}^{\infty} \frac{2 x}{\left(4 t^{2}+x^{2}\right)^{2}} d x+\mathcal{O}\left(\frac{1}{t^{4}}\right) \\
& =\frac{1}{4 t^{2}} \int_{0}^{\infty} \frac{2 x}{\left(x^{2}+1\right)^{2}} d x+\mathcal{O}\left(\frac{1}{t^{4}}\right)=\frac{1}{4 t^{2}}+\mathcal{O}\left(\frac{1}{t^{4}}\right)
\end{aligned}
$$

From this we deduce

$$
\mathcal{M}_{\kappa, i t}(1) \ll Q^{2 \kappa} e^{-\pi t}, \quad \mathcal{M}_{\kappa, i t}(2) \ll Q^{2 \kappa+1} e^{-\pi t},
$$

and by (2),

$$
\mathcal{M}_{\kappa, i t}(n) \ll_{n} Q^{2 \kappa+n-1} e^{-\pi t}, \quad \text { for all } n \in \mathbb{N} .
$$

Since $\left|\mathcal{M}_{\kappa, i t}(s)\right| \leq \mathcal{M}_{\kappa, i t}(\sigma)$ for all $\sigma>0$ we can use (i) of our lemma to deduce

$$
\mathcal{M}_{\kappa, i t}(s) Q^{-s-1-2 \kappa} e^{\pi t} \leq M, \quad M>0,
$$

on the sides of the rectangle given by the points $1 \pm 2 i t$ and $1000 \pm 2 i t$. By the maximum modulus principle this estimate holds for $s=1+\delta$ for some small $\delta>0$ and hence by (2),

$$
\mathcal{M}_{\kappa, i t}(\delta) \ll \delta^{-1} Q^{\delta-1-2 \kappa} e^{-\pi t}
$$

and so (6) is still valid on the sides of the rectangle given by the points $\delta \pm 2 i t$ and $1000 \pm 2 i t$, which implies (ii).

As a consequence of this lemma we obtain

Lemma 2. With the notation as before we have for $\delta \leq \sigma \leq 1-\delta$, 
(i) if $|\tau| \leq Q_{k}$ then

$\mathbf{C}_{k}( \pm \sigma+i \tau) \ll \delta^{-2}(1+|\tau|)^{2 \mp 2 \sigma}\left(\left(Q_{k}+|\tau|\right)\left(Q_{k}+1-|\tau|\right)\right)^{1 / 2 \mp \sigma}\left(\begin{array}{cc}1 & Q_{k}^{-2 r} \\ Q_{k}^{2 r} & 1\end{array}\right) ;$

(ii) if $|\tau|>Q_{k}$ then

$\mathbf{C}_{k}( \pm \sigma+i \tau)=\left(\frac{|\tau|}{\sqrt{2} \pi}\right)^{2 \mp 4 \sigma}$

$\times\left(\begin{array}{cc}e^{i g_{1}(s)}+e^{i g_{2}(s)}+\mathcal{O}\left(\left(1+\frac{Q_{k}}{\sqrt{|\tau|}}\right)^{2}\right) & \mathcal{O}\left(Q_{k}^{-2 r} e^{-\pi\left|\tau-2 \operatorname{Re}\left(t_{k}\right)\right|}\right) \\ \mathcal{O}\left(Q_{k}^{2 r} e^{-\pi\left|\tau-2 \operatorname{Re}\left(t_{k}\right)\right|}\right) & e^{i g_{1}(s)}+e^{i g_{2}(s)}+\mathcal{O}\left(\left(1+\frac{Q_{k}}{\sqrt{|\tau|}}\right)^{2}\right)\end{array}\right)$

where $g_{1}(s)$ and $g_{2}(s)$ are linear combinations of $\tau \log |s+\nu|,\left(\tau \pm \operatorname{Re}\left(t_{k}\right)\right)$ $\times \log \left|s \pm i t_{k}+\nu\right|, \sigma+\nu$, where $\nu \in\left\{-2,-1,0, \frac{1}{2}, 1\right\}, \tau, \operatorname{Re}\left(t_{k}\right)$ and some constant $c$.

Proof. Without loss of generality, assume $\tau>0$. Let us first look more closely at $\operatorname{det} \mathbf{M}_{k}(s)$. In [4] we have shown that

$$
\operatorname{det} \mathbf{M}_{k}(s)=\frac{\Gamma\left(s+2 i t_{k}\right) \Gamma\left(s-2 i t_{k}\right)}{s} \text {. }
$$

Furthermore, by (2),

$$
\begin{aligned}
c_{x y, k}(s) \\
=\mathcal{M}_{x, k}(1+s) \mathcal{M}_{y, k}(1-s)+\delta(x, y)\left(s^{2}+t_{k}^{2}\right) \mathcal{M}_{x, k}(s) \mathcal{M}_{y, k}(-s) \\
=\mathcal{M}_{x, k}(1+s) \mathcal{M}_{y, k}(1-s)+\delta(x, y) \mathcal{M}_{x, k}(s) \\
\quad \times\left(\frac{s-1}{s} \mathcal{M}_{y, k}(2-s)-\frac{r(2 s-1)}{s} \mathcal{M}_{y, k}(1-s)\right) .
\end{aligned}
$$

From (7) we see that $c_{ \pm \pm, k}(1-s)=s c_{ \pm \pm, k}(s) /(s-1)$, while from (3) it is obvious that $c_{ \pm \pm, k}(s)=c_{ \pm \pm, k}(-s)$. So we can conclude that $s c_{ \pm \pm, k}(s)$ has period one. Now we see from (2) and the subsequent remark that

$$
s c_{ \pm \pm, k}(s) \sin \left(\pi\left(s-2 i t_{k}\right)\right) \sin \left(\pi\left(s+2 i t_{k}\right)\right) \sin ^{2}(\pi s)=: T(s)
$$

is an entire function. Since this function also has period one, and since from the preceding lemma we also know that for $\tau \rightarrow \infty, s c_{x y, k}(s) \ll_{k} \tau^{2} e^{-\pi \tau}$, hence $T(s) \ll_{k} \tau^{2} e^{3 \pi \tau}$, we can expand $T(s)$ for $\tau>0$ in a Fourier series of the form $\sum_{n \geq-1} a_{k}(n) e^{2 \pi i n s}$. (The coefficients $a_{k}(n), n<-1$, must vanish because of the growth estimate for $T(s)$.) Also

$$
\sum_{n \geq-1}\left|a_{k}(n)\right|^{2}=\int_{0}^{1}|T(\sigma)|^{2} d \sigma \ll Q_{k}^{ \pm 4 r} e^{4 \pi \operatorname{Re}\left(t_{k}\right)}
$$

because of Lemma 1(ii). So finally, by Schwarz's inequality,

$$
T(s) \ll Q_{k}^{ \pm 2 r} e^{2 \pi\left(\tau+\operatorname{Re}\left(t_{k}\right)\right)}
$$


and consequently

$$
s c_{ \pm \pm, k}(s) \ll Q_{k}^{ \pm 2 r} e^{-\pi\left(\left|\tau-2 \operatorname{Re}\left(t_{k}\right)\right|+\tau\right)}, \quad|\tau|>Q_{k} .
$$

The statements of the lemma now follow from Stirling's formula and the preceding lemma.

\section{Proof of the theorems}

Proof of Theorem 1. From Lemma 2 we obtain

$$
R_{ \pm, k}^{*}(-\delta+i \tau) \ll \delta^{-2}(1+|\tau|)^{2+4 \delta} Q_{k}^{1+2 \delta} S_{ \pm, k}(1+\delta) .
$$

Use the Phragmen-Lindelöf principle to conclude the proof.

Proof of Theorem 2. The proof is based on a method of Landau (cf. [2]). But as we are in a slightly different situation (the $\Gamma$-factors in Landau's situation are now replaced by the $\mathcal{M}_{\kappa, i t}(s)$ ) we cannot merely refer to his results but have to redo his computations in part.

We define

$$
B_{ \pm, k}(x):=\sum_{j=1}^{m} \sum_{d>0} \sum_{\substack{d^{\prime}>0 \\ d^{\prime} \equiv \pm \alpha_{j} \bmod 1 \\ 0<d d^{\prime} \leq x}} b(d)\left|\varrho_{j, k}\left( \pm d^{\prime}\right)\right|^{2} d^{\prime},
$$

where $b(n)=1$ if $n$ is a square and 0 otherwise, and $x$ is as in the definition of $A_{ \pm, k}(x)$. Now it is clear that $B_{ \pm, k}(x)=\sum_{0<d \leq x} b(d) A_{ \pm, k}(x / d)$, and from this it follows by generalized Möbius inversion that

$$
A_{ \pm, k}(x)=\sum_{0<d \leq x} \mu(\sqrt{d}) b(d) B_{ \pm, k}(x / d),
$$

since $\mu(\sqrt{d}) b(n)$ is the Dirichlet inverse of $b(n)$. One should notice that for $\operatorname{Re}(s)>1$ we can write

$$
R_{ \pm, k}^{*}(s)=\sum_{j=1}^{m} \sum_{\substack{n \in \mathbb{N}_{0} \\ n \pm \alpha_{j}>0}} \frac{e_{j, k}( \pm n, s)}{n^{s}}
$$

with

$$
e_{j, k}( \pm n, s)=\sum_{d^{2} \mid n}\left(1 \pm \alpha_{j} \frac{d^{2}}{n}\right)^{-s}\left|\varrho_{j, k}\left( \pm \frac{n}{d^{2}}\right)\right|^{2}\left(\frac{n}{d^{2}} \pm \alpha_{j}\right) .
$$

Observe that $e_{j, k}(n, s) n^{-s}$ is still meaningful for $n=0$ and gives $\left|\varrho_{j, k}(0)\right|^{2}$ $\times \zeta(2 s)\left( \pm \alpha_{j}\right)^{1-s}$. 
Following Landau we introduce for $0<z<x$ the difference operator of degree $g$, acting on any function $h: \mathbb{R} \rightarrow \mathbb{C}$ via

$$
\Delta_{z} h(x)=\sum_{k=0}^{g}(-1)^{k}\left(\begin{array}{l}
g \\
k
\end{array}\right) h(x+(g-k) z) .
$$

For the rest of the proof we choose $g=4$ and one easily checks that (9)

$$
\frac{\Delta_{z} x^{s+4}}{(s+1)(s+2)(s+3)(s+4)}=\int_{x}^{x+z} \int_{x_{1}}^{x_{1}+z} \int_{x_{2}}^{x_{2}+z} \int_{x_{3}}^{x_{3}+z} x_{4}^{s} d x_{4} d x_{3} d x_{2} d x_{1} \text {. }
$$

This gives, for integer $l>3$,

$$
\Delta_{z} x^{l}=z^{4} l \ldots(l-3) x^{l-4}+\mathcal{O}\left(z^{l} x^{l-5}\right) .
$$

Now, using Perron's integral representation

$$
\begin{aligned}
& \Delta_{z}\left(\frac{1}{2 \pi i} \int_{(2)} \frac{x^{s+4}}{s(s+1)(s+2)(s+3)(s+4)} R_{ \pm, k}^{*}(s) d s\right) \\
& \quad=\int_{x}^{x+z} \int_{x_{1}}^{x_{1}+z} \int_{x_{2}}^{x_{2}+z} \int_{x_{3}}^{x_{3}+z} B_{ \pm, k}\left(x_{4}\right) d x_{4} d x_{3} d x_{2} d x_{1}=: \mathcal{B}_{ \pm}(x, z) .
\end{aligned}
$$

We restrict ourselves to the + case, the - case being completely analogous. We shift the line of integration in the integral on the left hand side of (10) to the line $\sigma=-1 / 4$, use the functional equation for $R_{+, k}(s)$ and then take the summation coming from the Dirichlet series outside the integral. Then push the line of integration further to $\sigma=-5 / 8+\varepsilon$ for some small $\varepsilon>0$ to see that the left hand side of (10) is

$$
\begin{aligned}
& \Delta_{z}\left(\frac{x^{4}}{24} R_{+, k}^{*}(0)+\frac{\pi^{2} x^{5}}{720} b_{+, k}\right) \\
& +\sum_{j=1}^{m} \sum_{n>0} \frac{1}{2 \pi i} \int_{(-5 / 8+\varepsilon)} n^{s-1}\left(\mathbf{C}_{11, k}(s) e_{j, k}(n, 1-s)\right. \\
& \left.\quad+\mathbf{C}_{12, k}(s) e_{j, k}(-n, 1-s)\right) \frac{\Delta_{z} x^{s+4}}{s(s+1)(s+2)(s+3)(s+4)} d s,
\end{aligned}
$$

where $\mathbf{C}_{i j, k}(s)$ are the entries of the transformation matrix $\mathbf{C}_{k}(s)$. From Lemma 2, in connection with [2], p. 221, we see that the integral converges. Lemma 2 further shows that the integral is

$$
\begin{aligned}
& \int_{|\operatorname{Im}(s)| \leq Q_{k}}+\int_{|\operatorname{Im}(s)|>Q_{k}} \\
& \ll_{\varepsilon} Q_{k}^{9 / 4+\varepsilon} x^{27 / 8+\varepsilon}\left(\frac{e_{j, k}(n, 13 / 8+\varepsilon)+Q_{k}^{-2 r} e_{j, k}(-n, 13 / 8+\varepsilon)}{n^{13 / 8}}\right)=: s_{1}(n) .
\end{aligned}
$$


We follow Landau and evaluate the integral in (11) in a different way. We use (9) and shift the line of integration to $\sigma=3 / 8+\varepsilon$. Notice that $\mathbf{C}_{i j, k}(s) / s$ is holomorphic at $s=0$, so we do not pass a pole and the integral in (11) becomes

$$
\begin{array}{r}
\frac{1}{2 \pi i} \int_{x}^{x+z} \int_{x_{1}}^{x_{1}+z} \int_{x_{2}}^{x_{2}+z} \int_{x_{3}}^{x_{3}+z}\left(\int _ { ( 3 / 8 + \varepsilon ) } \frac { ( x _ { 4 } n ) ^ { s } } { n s } \left(\mathbf{C}_{11, k}(s) e_{j, k}(n, 1-s)\right.\right. \\
\left.\left.+\mathbf{C}_{12, k}(s) e_{j, k}(-n, 1-s)\right) d s\right) d x_{4} d x_{3} d x_{2} d x_{1} .
\end{array}
$$

Again we write the integral over $s$ as $\int_{|\operatorname{Im}(s)| \leq Q_{k}}+\int_{|\operatorname{Im}(s)|>Q_{k}}$ and see that the entire fivefold integral is

$$
\ll_{\varepsilon} Q_{k}^{3 / 2+\varepsilon} x^{3 / 8+\varepsilon} z^{4} n^{-5 / 8}\left(e_{j, k}\left(n, \frac{5}{8}+\varepsilon\right)+Q_{k}^{-2 r} e_{j, k}\left(-n, \frac{5}{8}+\varepsilon\right)\right)=: s_{2}(n) .
$$

A first result is

$$
\begin{aligned}
\mathcal{B}_{+}(x, z)=\frac{\pi^{2}}{6} z^{4} x b_{+, k}+\mathcal{O}\left(z^{5} b_{+, k}\right. & \left.+z^{4} R_{+, k}(0)\right) \\
& +\mathcal{O}_{\varepsilon}\left(\sum_{j=1}^{m} \sum_{n \leq N} s_{1}(n)+\sum_{j=1}^{m} \sum_{n>N} s_{2}(n)\right) .
\end{aligned}
$$

Now observe first that we can replace $5 / 8+\varepsilon$ and $13 / 8+\varepsilon$ in $e_{j, k}( \pm n, \cdot)$ by $1+\delta, \delta>0$, without making a considerable error. We then choose $N=z^{-4} x^{3} Q_{k}^{3 / 4}$ for $z<x^{3 / 4} Q_{k}^{3 / 16}$, giving

$\sum_{j=1}^{m} \sum_{n \leq N} s_{1}(n)+\sum_{j=1}^{m} \sum_{n>N} s_{2}(n) \ll_{\varepsilon} x^{3 / 2+\varepsilon} Q_{k}^{57 / 32+\varepsilon} z^{5 / 2} M_{+, k}(1+\delta)\left(x Q_{k}\right)^{3 \delta}$.

Remark that $z^{4} B_{+, k}(x) \leq \mathcal{B}(x, z) \leq z^{4} B_{+, k}(x+4 z) \leq \mathcal{B}(x+4 z, z)$, which follows from (9), choose $z=x^{3 / 5} Q_{k}^{3 / 16}$ and use Theorem 1 for $R_{+, k}(0)$ to arrive at

$$
B_{+, k}(x)=\frac{\pi^{2}}{6} b_{+, k} x+\mathcal{O}_{\varepsilon}\left(Q_{k}^{3 / 2+\varepsilon} x^{3 / 5+\varepsilon} S_{+, k}(1+\delta)\left(x Q_{k}\right)^{3 \delta}\right) .
$$

A routine calculation together with $\delta=\left(\log x Q_{k}\right)^{-1}$ gives the desired result for $A_{+, k}(x)$.

\section{References}

[1] H. Daven port, Multiplicative Number Theory, Markham, Chicago, 1967.

[2] E. Landau, Über die Anzahl der Gitterpunkte in gewissen Bereichen, II, Nachr. Ges. Wiss. Göttingen (1915), 209-243.

[3] W. Magnus, F. Oberhettinger and R. P. Soni, Formulas and Theorems for the Special Functions of Mathematical Physics, Springer, 1966. 
[4] R. Matthes, Rankin-Selberg method for real analytic cusp forms of arbitrary real weight, Math. Z. 211 (1992), 155-172.

[5] - Prime geodesic theorem for the theta-case, J. Reine Angew. Math., to appear.

[6] R. A. Rankin, Contributions to the theory of Ramanujan's function $\tau(n)$ and similar arithmetical functions, Proc. Cambridge Philos. Soc. 35 (1939), 357-372.

[7] W. Roelcke, Das Eigenwertproblem der automorphen Formen in der hyperbolischen Ebene I, Math. Ann. 167 (1966), 292-337.

[8] -, Das Eigenwertproblem der automorphen Formen in der hyperbolischen Ebene II, ibid. 168 (1967), 261-324.

[9] A. Selberg, Bemerkungen über eine Dirichletsche Reihe, die der Theorie der Modulformen eng verbunden ist, Arch. Math. Naturvid. 43 (1940), 47-50.

FACHBEREICH MATHEMATIK

GESAMTHOCHSCHULE KASSEL UNIVERSITÄT

POSTFACH 101380

3500 KASSEL

GERMANY 